\title{
STATIONARY PLANS NEED NOT BE UNIFORMLY ADEQUATE FOR LEAVABLE, BOREL GAMBLING PROBLEMS
}

\author{
D. BLACKWELL AND S. RAMAKRISHNAN
}

\begin{abstract}
There exists a leavable, Borel gambling problem with a goal, where at most three gambles are available at each fortune, where each gamble has at most two points in its support, but for which stationary plans are not uniformly adequate.
\end{abstract}

Introduction. In the theory of gambling formulated by Dubins and Savage [5], a gambling problem is a triple $(F, \Gamma, u)$, where (i) the set of fortunes $F$ is a nonempty set, (ii) the gambling house $\Gamma$ is a subset of $F \times P(F)$, where $P(F)$ denotes the collection of all finitely additive probabilities defined on all subsets of $F$, and for each $f \in F$, the set of gambles available at $f, \Gamma(f)=\{\gamma:(f, \gamma) \in \Gamma\}$ is assumed to be nonempty, and (iii) the utility function $u$ is a real-valued function on $F$. For $f \in F$, a strategy $\sigma$, available at $f$, specifies (a) a gamble $\sigma_{0} \in \Gamma(f)$, and (b) for each positive integer $n$ and every sequence $\left(f_{1}, \ldots, f_{n}\right)$ of elements of $F$, a gamble $\sigma_{n}\left(f_{1}, \ldots, f_{n}\right) \in \Gamma\left(f_{n}\right)$. Roughly speaking, such a strategy, $\sigma$, available at $f$, specifies for a gambler with initial fortune $f$, (a) what he should do initially, and (b) what he should do after $n$ days, given that his fortunes on the first $n$ days were $\left(f_{1}, \ldots, f_{n}\right)$. A strategy $\sigma$ available at $f$ induces a probability, which we shall denote once again by $\sigma$, defined on the Borel subsets of the history space $H=F^{N}$, where $N$ denotes the set of positive integers and $H$ is equipped with the product of discrete topologies. Let $\Sigma(f)=\{\sigma$ : available at $f\}$. The value of a gambling problem $V$ is a real-valued function on $F$, defined by

$$
V(f)=\sup _{\sigma \in \Sigma(f)} \int \limsup _{n} u\left(x_{n}\right) d \sigma(h), \quad f \in F,
$$

where $h=\left(x_{1}, \ldots, x_{n}, \ldots\right)$ is an element of $H$. (We only consider gambling problems for which $\int \lim \sup _{n} u\left(x_{n}\right) d \sigma(h)$ is defined for all $f \in F$ and $\sigma \in \Sigma(f)$.)

$A$ stationary plan $S$ is a function defined on $F$ into $P(F)$ such that $S(f) \in \Gamma(f)$ for all $f \in F$. A stationary plan defines, for each $f \in F$, a strategy $S_{f}$ available at $f$. The initial gamble of $S_{f}$ is $S(f)$ and, given that the fortunes on the first $n$ days were $\left(f_{1}, \ldots, f_{n}\right)$, the gamble specified by $S_{f}$ is $S\left(f_{n}\right)$. The utility of a stationary plan $S$ is a real-valued function $U_{S}$ on $F$, defined by

$$
U_{S}(f)=\int \underset{n}{\limsup } u\left(x_{n}\right) d S_{f}(h), \quad f \in F,
$$

Received by the editors September 16, 1986 and, in revised form, January 15, 1987.

1980 Mathematics Subject ('lassification (1985 Revision). Primary 60G40.

Key words and phrases. Gambling problem, leavable, Borel measurable gambling problem, stationary plan, uniform adequacy. 
where, as before, $h=\left(x_{1}, \ldots, x_{n}, \ldots\right)$ is an element of $H$. We say that stationary plans are uniformly adequate for the gambling problem $(F, \Gamma, u)$ if for every $\varepsilon>0$, there exists a stationary plan $S$ (possibly depending on $\varepsilon$ ) such that

$$
V(f)-U_{S}(f)<\varepsilon \text { for all } f \in F .
$$

A gambling problem $(F, \Gamma, u)$ is called leavable if $\delta_{f} \in \Gamma(f)$ for all $f \in F$, where $\delta_{f}$ denotes the point mass at $f$. Roughly speaking, in a leavable problem, the gambler has the option of quitting whenever he wishes, taking with him his current fortune at that time. A gambling problem with a goal is a gambling problem where the utility function assumes only two values, 0 and 1 . The goal $G$ for such a problem is the collection of all those fortunes whose utility is 1 . In this case, $V$ is the best chance of being in $G$ infinitely often. (In the leavable case this is the same as the best chance of being in $G$ some time.)

If $(F, B)$ is a standard Borel space (i.e. $F$ is a Borel subset of a complete, separable, metric space, and $B$ is the $\sigma$-algebra of its Borel subsets), we shall denote by $M$, the collection of all countably additive probabilities on $(F, B)$, and by $B^{*}$, the smallest $\sigma$-algebra of subsets of $M$ such that, for each $A \in B$, the map $\mu \rightarrow \mu(A)$ on $M$ is measurable.

A gambling problem $(F, \Gamma, u)$ is called Borel if

(i) $(F, B)$ is a standard Borel space, $u$ is Borel measurable,

(ii) for each $f \in F$ and $\gamma \in \Gamma(f), p(\gamma) \in M$, where $p(\gamma)$ denotes the restriction of $\gamma$ to $B$, and

(iii) $\{(f, p(\gamma)): f \in F, \gamma \in \Gamma(f)\}$ belongs to the product $\sigma$-algebra $B \otimes B^{*}$.

Ornstein [8] proved that for a leavable gambling problem, if $F$ is countable (and all gambles are countably additive), stationary plans are uniformly adequate. He also showed that there is a leavable gambling problem with $F$ uncountable for which stationary plans are not uniformly adequate. In his example, the cardinality of $F$ is greater than that of the continuum and hence the gambling problem is not Borel. Whether stationary plans are uniformly adequate for leavable, Borel gambling problems has been open for a number of years. (See [7].) In this paper we settle this question with the following

THEOREM. There exists a leavable, Borel gambling problem with a goal, where at most three gambles are available at each fortune, and where each gamble has at most two points in its support, but for which stationary plans are not uniformly adequate.

REMARKS. For gambling problems which are not necessarily leavable, good stationary plans need not exist even if $F$ is finite. (See Chapter 3, §9 of [5] for examples.) Dubins and Sudderth [6] and Pestien [9] proved the existence of gambling problems, with the fortune set having cardinality $\aleph_{1}$, for which stationary plans are not uniformly adequate. However, they allowed finitely additive gambles that are not countably additive. Dubins and Sudderth [7] showed that for leavable, Borel gambling problems (in fact, more generally for leavable, analytic gambling problems), stationary plans are 'almost uniformly adequate' (see [7, Proposition 7.1]). This, in particular, shows 'adequacy' of stationary strategies for such problems. (See [7] for relevant definitions.) Some of the positive results on uniformly adequacy can be found in $[2,3$ and 7$]$. 
ProOF OF THE THEOREM. Let $T$ denote the tree of all finite sequences of positive integers, including the empty sequence $e$. Let $L$ be the set of all functions $l$ from $T$ to the nonnegative integers. A function $l$ in $L$ may be thought of as a labeled tree: the tree $T$ with a nonnegative integer label on each vertex. The set of fortunes $F$ for our gambling problem will be $L \cup\{g\} \cup\{b\}$, where $g$ and $b$ are not in $L$, and $g \neq b$.

The gambles available are as follows:

$\Gamma(g)=\left\{\delta_{g}\right\}$ and $\Gamma(b)=\left\{\delta_{b}\right\}$.

For $l \in L$ with $l(e)=0, \Gamma(l)=\left\{\delta_{l}, \delta_{g}\right\}$.

For $l \in L$ with $l(e)=n(\geq 1), \Gamma(l)=\left\{\delta_{l}, \delta_{l^{\prime}},(1-1 / n) \delta_{l \circ n}+\delta_{b} / n\right\}$

where $l^{\prime}$ is the element of $L$ defined by $l^{\prime}(e)=n+1$ and $l^{\prime}(t)=l(t)$, for $t \neq e$; and $l \circ n$ is the element of $L$ defined by $l \circ n(t)=l(n t), t \in T$, where $n t$ is the finite sequence of positive integers starting with $n$ and followed by those in $t$.

The utility function $u$ is 1 at $g$ and 0 at every other fortune.

For this gambling problem, it is easily checked that, for every strategy $\sigma$ available at $f, \int \lim \sup _{n} u\left(x_{n}\right) d \sigma(h)$ equals the probability of reaching $g$ starting from $f$ and using $\sigma$, and hence $V(f)=$ best chance of reaching the goal $g$, starting from the fortune $f$.

For $t \in T$ and a nonnegative integer $k$, let $A(t, k)=\{l \in L: l(t)=k\}$. The collection of sets $\{A(t, k): t \in T, k \geq 0\} \cup\{\{g\},\{b\}\}$ forms a subbase for a topology on $F$ (under this topology, $g$ and $b$ are isolated points and the relative topology on $L$ is the topology of pointwise convergence). If $B$ denotes the $\sigma$-algebra of Borel subsets of $F$, plainly $(F, B)$ is a standard Borel space. To complete the verification that the above gambling problem $(F, \Gamma, u)$ is a leavable, Borel gambling problem with a goal, we only need to check condition (iii) in the definition of a Borel gambling problem. This can be done, for example, by showing that the map $\varphi_{i}$ on $F$ into $M$ is measurable, $i=1,2,3$, where

$$
\begin{aligned}
\varphi_{1}(f) & =\delta_{f} \quad \text { for all } f \in F, \\
\varphi_{2}(b)=\delta_{b}, \quad \varphi_{2}(g)=\delta_{g}, \quad \varphi_{2}(l) & = \begin{cases}\delta_{g} & \text { if } l(e)=0, \\
\delta_{l^{\prime}} & \text { if } l(e) \geq 1,\end{cases} \\
\varphi_{3}(b)=\delta_{b}, \quad \varphi_{3}(g)=\delta_{g}, & \varphi_{3}(l)= \begin{cases}\delta_{g} & \text { if } l(e)=0, \\
(1-1 / n) \delta_{l \circ n}+\delta_{b} / n & \text { if } l(e)=n(\geq 1) .\end{cases}
\end{aligned}
$$

This verification is routine using well-known results on measurability of sets of measures which can be found in [ 1 or $\mathbf{4}]$.

Let $C$ denote the set of all fortunes $l \in L$ such that every infinite path down the tree must hit a zero, i.e. for every sequence $t(1), t(2), \ldots$ of positive integers, there is a $k \geq 1$ with $l(t(1), \ldots, t(k))=0$. The proof of the Theorem will be completed by verifying the following two claims.

Claim 1. $V(l)=1$ for every $l \in C$.

Claim 2. For any stationary plan $S$ and any $\varepsilon>0$, there exists a fortune $l \in C$ such that $U_{S}(l)<\varepsilon$.

To justify Claim 1 , suppose $V(l)<1$. We show that $l \notin C$ by finding an infinite path $t(1), t(2), \ldots$ that avoids all zeros of $l$. Let $t(1)$ be the least positive integer $n$ with the property that $V(l \circ n)<1$. All large $n$ have the property, since by first 
increasing $l(e)$ to $n$ and then attempting to move to fortune $l \circ n$, we can reach $g$ with probability at least

$$
a(n)=(1-1 / n) V(l \circ n)-1 / n,
$$

and since $a(n) \leq V(l)$, it follows that $V(l \circ n)<1$ for all large $n$. We can apply the same argument repeatedly to get $t(1), t(2), \ldots$ with $V\left(l^{(k)}\right)<1$ for all $k$, where $l^{(k)}(t)=l(t(1), \ldots, t(k), t)$ for $t \in T$. Since $V\left(l^{(k)}\right)<1$, we must have $l^{(k)}(e)>0$, i.e. $l(t(1), \ldots, t(k))>0$.

To establish Claim 2, suppose $l_{1}, l_{2}, \ldots$ is a sequence of fortunes in $C$ with $\inf U_{S}\left(l_{n}\right)=\alpha>0$. We find an $l \in C$ such that $U_{S}(l)<\alpha$. For each $k \geq 1$, let $f_{k}$ be equal to $l_{n}$, where $n$ is least integer such that $U_{S}\left(l_{n}\right)<(1+1 / k) \alpha$.

Define $l$ by $l(e)=1, l(k t)=f_{k}(t), t \in T$. Then $l \in C$ and, for some $k$,

$$
U_{S}(l)=(1-1 / k) U_{S}\left(f_{k}\right) \leq\left(1-1 / k^{2}\right) \alpha<\alpha .
$$

Thus, it follows that $\inf _{f \in C} U_{S}(f)=0$. This finishes the proof of the Theorem.

\section{REFERENCES}

1. D. P. Bertsekas and S. E. Shreve, Stochastic optimal control: the discrete time case, Academic Press, New York, 1978.

2. C. Dellacherie, Les sous-noyaux élémentaires, Théorie du Potentiel Proceedings (Orsay, 1983), Lecture Notes in Math., vol. 1096, Springer-Verlag, Berlin and New York, 1983, pp. 183-222.

3. C. Dellacherie and P. A. Meyer, Probabilités et potentiel, théorie discrete du potential, Hermann, Paris, 1983.

4. L. E. Dubins and D. A. Freedman, Measurable sets of measures, Pacific J. Math. 14 (1964), 1211-1222.

5. L. E. Dubins and L. J. Savage, Inequalities for stochastic processes, Dover, 1976.

6. L. E. Dubins and W. D. Sudderth, An example in which stationary strategies are not adequate, Ann. Probab. 3 (1975), 722-725.

7. 476.

8. D. Ornstein, On the existence of stationary optimal strategies, Proc. Amer. Math. Soc. 20 (1969), 563-569.

9. V. C. Pestien, Inadequacy of stationary plans for finitely controlled gambling problems, Preprint, 198 ?.

Department of Statistics, University of California, Berkeley, California 94720

Department of Mathematics and Computer SCIEnCE, University of Miami, CORAL GABLES, Florida 33124 